\title{
A Complete Interoperable eHealth System Based on ZigBee Pro Standard
}

\author{
Iker Caballero $^{1}$, Javier Vicente ${ }^{1}$, Begoña García ${ }^{1} \&$ Amaia Méndez ${ }^{1}$ \\ ${ }^{1}$ DeustoTech-Life Unit, Deusto, Institute of Technology, University of Deusto, Bilbao, Spain \\ Correspondence: Amaia Méndez, DeustoTech-Life Unit, Deusto, Institute of Technology, University of Deusto, \\ Avda. de las Universidades, Bilbao, Spain. Tel: 34-944-139-000. E-mail: amaia.mendez@deusto.es
}

Received: November 14, 2011 Accepted: November 30, 2011 Online Published: June 1, 2012

doi:10.5539/nct.v1n1p79 URL: http://dx.doi.org/10.5539/nct.v1n1p79

This work was partially supported by the Basque Country Department of Education, Universities and Research

\begin{abstract}
The eLife research group at University of Deusto is trying to socialize new technologies through projects focused on health. Telemedicine and eHealth systems are being used widely to improve health services or to develop value added ones. The main aim of this project is to design an eHealth system that increases life quality. It is focused on people who need daily support because of age, disability or disease. A wireless sensor network has been developed and it is integrated in a Set-Top-Box that allows controlling the network from TV. Besides, some devices have been designed so the article describes them as well as the software development.
\end{abstract}

Keywords: ZigBee Pro, eHealth, electronic design, wireless sensor network

\section{Introduction}

Biomedical Engineering and Information and Communication Technologies are growing together at high speed. The European Commission has been promoting them for two decades through its Framework Programmes (FP), which are focused on multiple topics. One of most important areas where both participate is telemedicine, better known nowadays as telehealth. In the FP6 (2002-2006) health research projects were priorities in many cases (Iakovidis, Le Dour, \& Karp, 2007). More specifically, personal eHealth systems and rehabilitation technologies can be found among the Information Society Technologies priorities, neurosciences and medical imaging in Life Sciences, Genomics, and Biotechnology for Health Programme, and biosensors as part of the Nanotechnologies Programme. The FP7 is organized into five different blocks encompassing both collaborative and individual group research (in the latter case, without need for internationalization). It also supports researcher mobility and career development. The Collaboration block, comprising two thirds of the total budget, is also divided into different topics. One of them is called Health and there are also topics related to nanotechnologies and ICTs, as in FP6.

Telemedicine (Krol, 1997) defined as the use of computer and communication technologies to provide healthcare services between distant locations, started by the sharing of information between medical centers. The first applications were focused on audio and video links between these locations in order to resolve problems all together if necessary, to establish remote consultations and to access common databases. Nowadays, with the rapid growth of technologies, a wide range of health services have been developed with the support of sizeable research programs. Common applications are based on image and signal processing, specialized medical systems or telecommunication standards, among others. There is also great support for wearable systems (Lymberis \& Dittmar, 2007; Chiu, 2006) and applications in order to complement eHealth and monitoring systems (McAdams, 2011).

Nevertheless, these eHealth advances have not been introduced into the market nor adopted by it. There are a lot of technological, legal or financial barriers (Paul, Pearlson, \& McDaniel, 2009; Gibbons, 2008) involved in this process, sometimes too difficult to resolve. In technological terms, we can encounter problems not only in the user needs (if technology can solve the particular problem) but also in the user capabilities (if end-user can manage new technologies and applications). It is important because the users' capabilities vary, and rural people are, on average, the least technologically proficient but the neediest of such services (Sudhahar, y otros, 2010). Technological barriers are cited as a significant cause of the low utilization rates of eHealth services, but a 
reduction of these barriers does not guarantee wider utilization since other barriers are as important as the technological ones.

There is also a disagrement over costs (Wells, 2002; Poon \& Zhang, 2008): whether eHealth systems cause a cost reduction or not (Nitzkin, 1996), and who such reduction would be for. We should realize that the population is aging (Mauksch, 1987), thus increasing the utilization rates of health services (Moullec \& Ray, 2009). The elderly is a collective that generates higher costs than the rest of the population. This idea, together with the ever increasing population, shows that health care costs are also increasing. The development of new prevention-oriented services can be translated into medium to long term investments (Iluyemi \& Briggs, 2009; Hui, Yanting, \& Xue, 2010). However, the inclusion of technology in the health sector implies a need for training (for both specialists and patients) due to possible deficiencies in technological knowledge. This failing results in the development of adaptive user interfaces (Vasilyeva, Pechenizkiy, \& Puuronen, 2005). Health services have to ensure not only accurate and up-to-date, but also easy to understand, information. These interfaces should take into account both content and navigation adaption. The contents should be adapted to the patients' situation, illness and treatment. Users could choose how to see the content, but it is the system that offers possibilities based on the specific patient.

Another important feature of eHealth services is the information shared through electronic medical records (Detmer, Bloomrosen, Raymond, \& Tang, 2008; De, 2005). As you can see, many factors contribute to the economic effects of eHealth systems, and thousands of hours could be spent discussing them. All these changes should be well organized with the aim of avoiding waste and inefficiency, so a better management must be achieved (Sheng, Hu, Wei, \& Ma, 1999). A well organized service reduces the number of treatment days (Akematsu \& Tsuji, 2009). If there are affordable systems for patients, the diagnostics and some tests could be performed at a patient's home without the need for new appointments using telematic services.

Apart from multimedia (Cabral \& Kim, 1996) and telematic services, wireless sensor networks (Yan, Huo, Xu, \& Gidlund, 2010) have a place in the end users' home. These networks provide the link between the user and the service itself. If mass adoption of eHealth systems is desired, not only is user adaption to the application needed, but also the standardization of systems with the aim of different services and devices operating together, in other words, promoting interoperability. Bear in mind that often standardization, and therefore interoperability, is contrary to the interests of manufacturers, but it encourages competitiveness. And this competitiveness means that prices fall. Also important is the collaboration of government agencies such as the European Telecommunications Standards Institute (ETSI) (Hine, Petersen, Pluke, \& Sund, 2008), as well as partnerships and alliances between companies (Piniewski, Muskens, Estevez, Carroll, \& Cnossen, 2010). On one hand, the ETSI proposes the standardization of both application framework and architecture. On the other hand, Continua Alliance has designed some guidelines for the standardization of health devices. There are also social features which are truly important, such as security. Health information is very sensitive and technology should be capable of maintaining its privacy. So, both technical and social factors must be analyzed for a strong standardization (Chadwick, 2007).

Although there are a lot of factors involved in the development of telehealth services, the main aim of this project is to try to promote standard-based systems in order to achieve interoperable applications for eHealth. This aim could increase the number of interoperable devices and thus competitiveness. Therefore, the prices of end users' systems will be reduced. In accordance with this aim, several specific goals can be highlighted:

- Design of a standard-based system to permit interoperability between devices and to reduce the final costs.

- Use of a single technology for healthcare devices and home automation.

- Design a gateway device in order to control the system from a PC user application.

\section{Methods}

It is not new that each wireless and wired technology has its own advantages and disadvantages. However, mobility is one of most important features in eHealth systems or other kinds of patient monitoring systems and it can only be provided by wireless technologies. Therefore, wireless technologies have gained much ground in the eHealth market. Another important feature is low power consumption, so the technology selected should be wireless and with reduced power consumption (Chien, Xu, \& Molloy, 2010). Bluetooth (in its low-energy version), ZigBee and ANT are technologies with these features. Each one has its advantages and disadvantages, in terms of number of nodes, topologies supported, etc. But it is difficult to point out one of them as the best or the favorite. There are a lot of reviews comparing them (Gomez \& Paradells, 2010), but it is quite clear that each one has its own market. 
Continua Alliance, mentioned above, has selected Bluetooth-LE and ZigBee for its second version of guidelines (Piniewski, Muskens, Estevez, Carroll, \& Cnossen, 2010). Bluetooth stands as the technology for mobile devices (e.g., a watch with an ECG monitor connected to the mobile phone), while ZigBee focuses toward low-power sensors (bed pressure sensors, fall detectors, etc).

The problem facing this choice is the need for platforms that support different technologies, thus adding complexity to the system. It is not a complexity in development, but for interoperability. It is difficult to achieve interoperability using a single technology, so adding different technologies makes it more difficult. In addition, it involves the development of gateways (Hwang, In, Park, \& Eom, 2003; Song, Zhou, Zhang, \& Song, 2008). These gateways are common elements used to interconnect different systems or technologies in order to provide a more complete service. Thus, indoor sensor networks can be connected to telematic systems to send or record information (Farshchi, Pesterev, Nuyujukian, Mody, Judy, \& Bi-Fi, 2007), and also to control or manage the network from remote sites. However, the inclusion of different technologies in the indoor system involves the development of gateways within the sensor network itself. For this reason, the project develops a platform based on a single technology.

The technology selected for this project was ZigBee, which provides the best performance for a single technology based system and it is based on IEEE802.15.4 standard (Bandyopadhyay, 2010). ZigBee adds network and routing services to IEEE802.15.4. ZigBee was created by the ZigBee Alliance, a non-profit association whose main aim is to develop standards suiting the real evolving needs of manufacturers and developers. The Alliance designed some application profiles with industrial experts to meet the market needs. Specifically, the Home Automation Profile offers a global standard for smart homes, enabling the control of lights, environment or security among others. There is also a Health Care Profile, which enables reliable monitoring of non-critical healthcare services, targeted as independent life, health or wellness. Nowadays the focus is the end user, that is the customer, and they are demanding standard systems that can operate together, in order not to fill the house with numerous independent systems.

\subsection{ZigBee Basics and Wireless Devices}

ZigBee is a new technology that provides networking features over IEEE802.15.4 wireless standard. IEEE805.15.4 is a standard defined by the IEEE for low-rate wireless PANs. It defines the link and physical layer (low-power spread spectrum at $2.4 \mathrm{GHz}$ with a basic rate of $250 \mathrm{kbps}$ ) The ZigBee Alliance developed the rest of the layers with the collaboration of companies working in electronics. The Alliance has developed several releases of the protocol. The latest one is ZigBee 2007, more commonly known as ZigBee Pro.

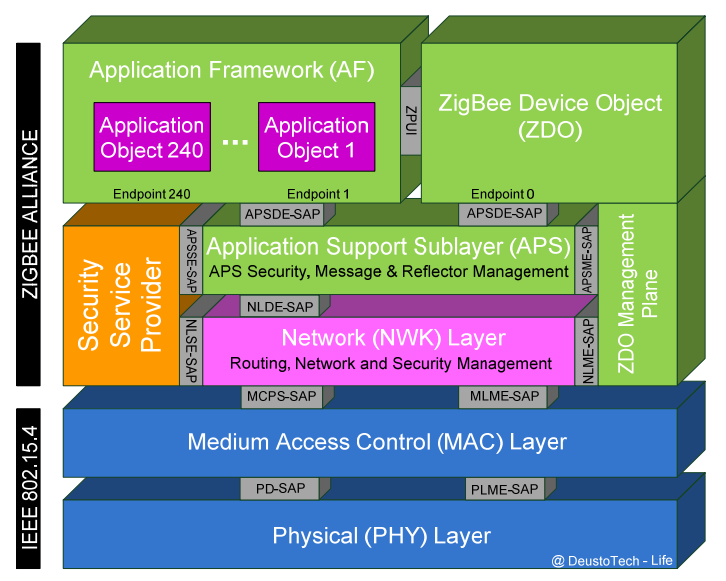

Figure 1. ZigBee stack

As it can be seen in Figure 1, there are some layers defined by the Alliance to support all features needed in a WPAN. The last change was the inclusion of a new part in the Application Framework (AF) layer: the application objects and the ZigBee Cluster Library. The AF layer provides some features for specific applications and devices, thus it can achieve interoperability and ubiquity of devices from multiple manufacturers, so that devices with same functionalities should behave similarly. Although this is a huge step into standardization and interoperability, the addition of this new feature brings about greater complexity in application development.

Good-quality research work has been done into this, because it is a really arduous task to understand how the 
stack is implemented. Each ZigBee device should have several functionalities and the same device could be a light switch and a TV volume control. For this reason, application objects have been developed. Each device could have up to 240 application objects, usually called endpoints. So, we can have an only device with two endpoints: one of them for TV volumen control, and the other one to switch the living-room lights on and off, for example. The previously mentioned ZCL has been developed with the purpose of providing specific functionalities for each device, so that a light-switch behave similarly regardless of the manufacturer that have design it. Due to the multiple applications that can be developed in this type of networks, the ZigBee Alliance has defined some profiles in order to sort the clusters (seen as the specific functions that a device could perform) according to subject and application, such as: Home Automation, Healthcare, Smart Energy, Telecom Services, etc.

The clusters could be defined as client (output) or server (input) clusters. Following the previous example, the light switch behaves like a client that wants to change the light value. This light is the server, where the action will be performed. The light-switch is going to have a client cluster (an output cluster) to perform the action of switching on/off a light. The light is going to have a server cluster (an input cluster) in order to receive the request from the switch. Each endpoint is associated to a unique profile, but not the only one that could have that associated profile (a device could have many endpoints associated to the same profile, with different or even the same clusters).

Another basic concept that has to be understood is the attributes of each cluster. The light device, which has the input cluster, must have an attribute that shows the current value of the light. In this case, it is a Boolean value, on or off. Each cluster has its own attributes, some of them are mandatory and others are optional. Only the server side has to keep the attributes.

As can be seen from above, the ZigBee Pro Stack has gained in complexity. There are many concepts and, although the specification is quite clear, is difficult to manage it. Profiles, clusters and attributes can easily be confused. Even so, the ZCL is a big step towards interoperability.

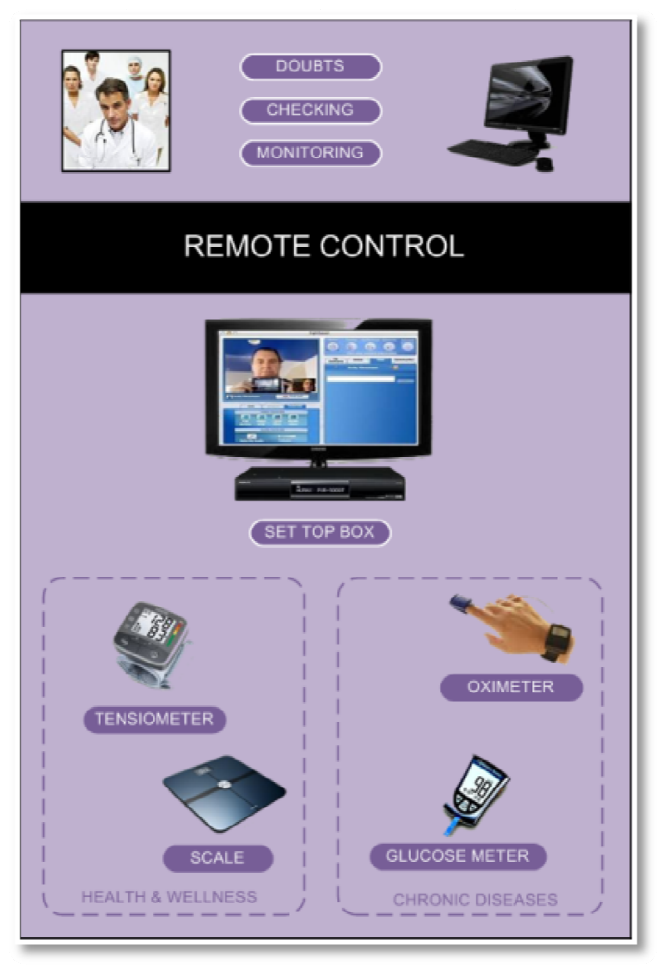

Figure 2. A complete eHealth system

\section{System Design}

The system that has been designed in this project is similar to that presented in Figure 2. A complete system with wireless sensors or devices attached to a Set-Top-Box, directly connected to professionals or patients' families. To achieve the proposed goals, two main parts have developed: the the ZigBee devices, and the design of new 
devices that are not available on the market, or devices that are not adapted to ZigBee. The Set-Top-Box is developed with an ARM processor running on an embedded Linux distribution. It is designed to be connected to a television set and manage the sensor network from it, without needing to install or have more systems or devices around the house.

All the devices have been developed with the JN5148 module from Jennic (now acquired by NXP). It is a 32-bit RISC microcontroller with low power consumption. It has a lot of peripherals, such as UARTs, ADC and DAC converters, DIO pins, etc. Jennic has also designed a ZigBee Protocol Stack certified by the ZigBee Alliance. Jennic provides a complete software solution for developing ZigBee compliant applications.

\subsection{Software Development}

Home Automation is the main profile used in this project. The developed clusters are included in the Home Automation specification of the ZigBee Alliance. They are divided into two sections: the general commands and the commands of specific clusters. The basic functions have been designed to discover, read or write the attributes of any cluster. However, the clusters have their specific commands. The clusters used in this project are as follows:

- Groups cluster, to discover, add or remove a device from a group.

- On/Off cluster, with its commands to switch on, switch off or toggle the light.

- Level Control cluster, to move up or down the level of a light, either continuously or in steps.

- Analog Input cluster, with no specific commands.

Along with these clusters, the development carried out includes the next devices:

1) On/Off Light Switch: Typical simple switches to change the current state of a light (switch on, switch off or toggle).

2) On/Off Light: A simple light, controlled by a light switch.

3) Dimmer Switch: A dimmer which can change the level of a dimmable light.

4) Dimmable Light: A dimmable light controlled by a dimmer switch.

5) Analog sensor: Any sensor that gives an analog value. In this project, a galvanic skin response meter.

To summarize and combine the concepts of ZigBee and the developed application devices, a table per device is presented in Figure 3 showing the information on them.

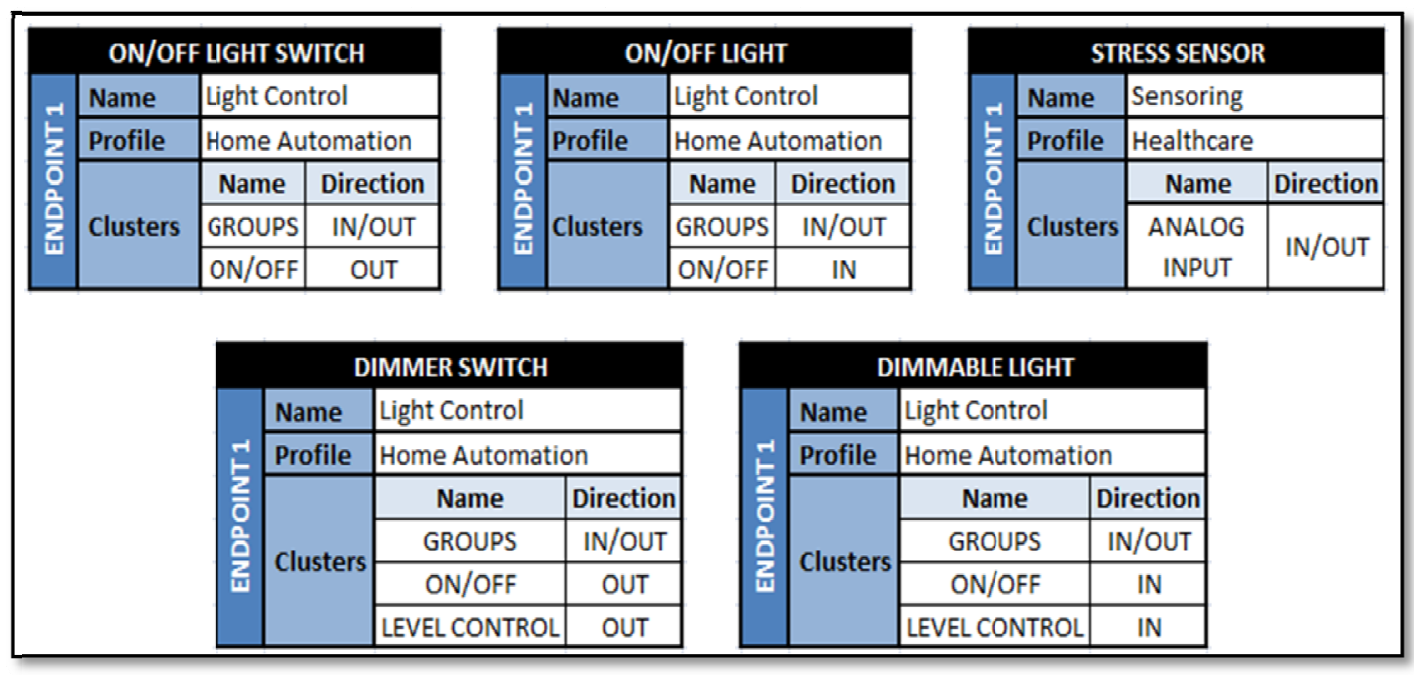

Figure 3. Zigbee information of devices

Another feature developed is the binding process between devices. A bind is a direct link between an input cluster from an application object to an output cluster of another application object, for example, a link between the output on/off cluster of a switch and the input on/off cluster of a light device. This link is made through a binding process. The devices ask for a binding and the parent device (a router or a coordinator) analyzes whether 
the clusters of both devices are compatible. If they are compatible, the parent responds with the address and the endpoint of the bound device (the other device, with a compatible cluster). This information is kept in the binding table of the device. When some data should be transmitted to the binding devices, the source device sends a frame per entry in the binding table. If a switch has three light entries in its binding table, it is able to control three lights independently if they are in the same or different devices (only one ZigBee radio). This link is called one-to-many binding (Figure 4a). It is also possible for one light to be controlled by several switches, commonly called many-to-one binding (Figure 4b). In this case, each switch adds the same table entry.
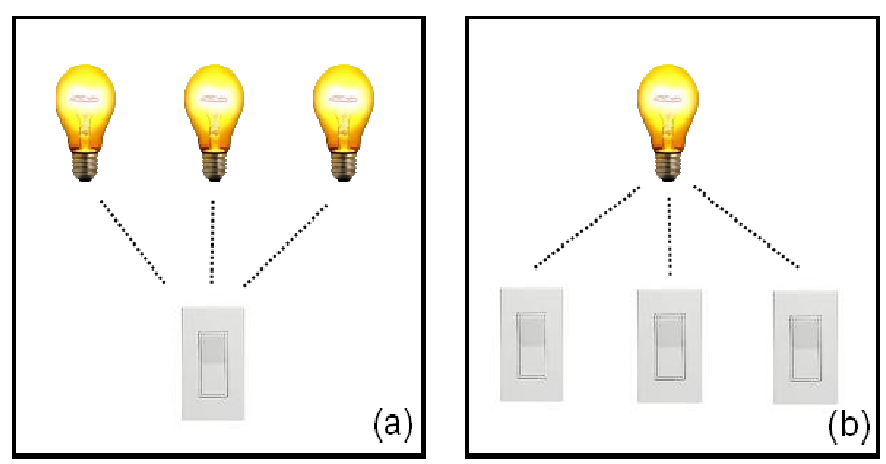

Figure 4. (a) One-to-many binding; (b) Many-to-one binding

This binding process is a point-to-point binding; in other words, you have to make a binding per pair of devices. If a switch has to control three lights, the binding process has to be carried out three times with the different lights and the same switch. However, there is another way to address the binding process.

As can be seen in the previous tables, all devices related to lighting have the Groups cluster. This has been developed with the intention of creating groups of lights, that is, a room could have numerous lights. By adding these independent lights to a group, we could light them up all together without having to switch on the lights one by one. The Groups cluster allows adding an endpoint to a group in order to receive all data addressed to the group in the specific endpoint. It also allows removing all groups or one specific group from the groups table, or asking for the groups an endpoint belongs to.

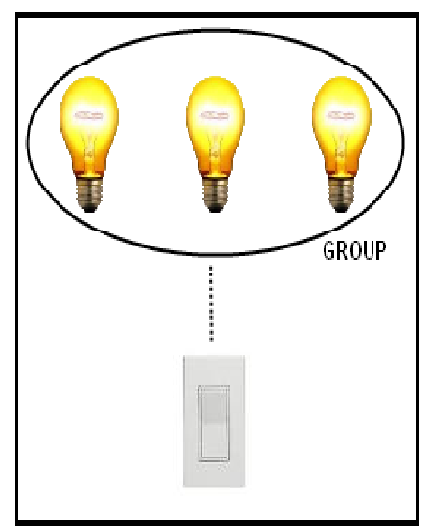

Figure 5. Group binding

In the present case, each device performs a different action when it receives a message to add to a group. On one hand, the light devices add the group to its groups table; so, when a switch-on command is sent to a group, it reaches all the devices included in that group lighting them up. On the other hand, when an add-group command is sent to a switch, it does not add the group ID to the group table but to the binding one. Thereby, when the switch sends the switch-on or switch-off command to the binding devices, it sends it to every group and every device stored in the binding table. This way of binding is called group binding, and is shown in Figure 5. When a device is bound to a group, there is a single entry in the table; there is not an entry for each device in the group. 


\subsection{Gateway Development}

Although the network can operate completely independently, the need to control it through an embedded system or a personal computer arises. Thereby, user applications based on any platform could be designed to manage the network. The proposed design is a gateway controller between the network coordinator and the computer. The communication channel is through a UART port of the microcontroller. The computer, meanwhile, establishes a virtual serial port, established through a USB port.

The protocol has been designed with AT-like commands. There are commands from general to the most basic kind. There are some commands used to discover information on the devices: how many endpoints they have their input and output clusters, etc. There are also specific commands according to the clusters developed. There are specific commands to add a device to a group, to send on/off commands to the lights, to read a specific attribute of a device, etc.

Table 1. Get simple descriptor command

\begin{tabular}{lc}
\hline Command & GSDS \\
Parameters & NWKADDR, EP ID \\
Format & GSDS=XXXX.XX \\
Example & GSDS $=6$ DB4.01 \\
\hline
\end{tabular}

Table 2. On/Off unicast command

\section{Command}

Parameters

Format

Example
OOUC

NWKADDR, SRC EP, DST EP, ON/OFF CLUSTER COMMAND

\section{OOUC $=$ XXXX.XX.XX.XX}

OOUC $=6147.02 .01 .02$

The command shown in Table 1 is used to obtain information on a device. The command requests the simple descriptor of a specific endpoint of the device. The descriptor contains plenty of information, such as clusters (both input and output), the profile associated to the endpoint, the device indetifier, and so on. It is a general command, not specific of a profile. The command shown in Table 2, however, is a specific cluster command. It corresponds to the On/Off cluster. The command is used to send a unicast frame to switch on, switch off or toggle a light of a device.

During the progress of this project, several devices have been designed in a parallel way. One example of them is the dimmable light device. This device is included in the Home Automation profile (according to ZigBee device profiles). The dimmable light device permits the user to modify the level of brightness in an incandescent light bulb. There are many ways of controlling this level, but we have chosen a digital control circuit.

The level of brightness is controlled by four general purpose output pins of the ZigBee module. The circuit also has a 555 timer and a 4-bit counter. The 555 timer works as the counter clock. The output pins of the microcontroller control the initial value of the counter. Thus, when a zero crossing of the AC signal is detected, it resets the counter. The counter starts from the initial value (provided by the microcontroller pins) to zero, allowing the current to pass through the bulb. Once the counter has reached zero, the borrow signal of the counter stops the current flow into the bulb. The process, allowing the power signal to pass through the bulb at a time interval during the half-cycle, controls the level of brightness. This current flow is controlled by an optotriac.

As the bulb has to be powered by the AC signal, this signal is also used for the rest of the circuit. We need to convert this AC signal into a DC signal, and lower its voltage. Therefore, in the complete schematic shown in Figure 6, a supply signal adaptation stage appears. This consists of a $9 \mathrm{~V}$ transformer and a rectifier bridge. The transistors in the center of the figure are used for the zero crossing detector system. The top of the image is another adaptation stage. We need to reduce the voltage from $9 \mathrm{~V}$ to the $3.3 \mathrm{~V}$ needed by the microcontroller. 


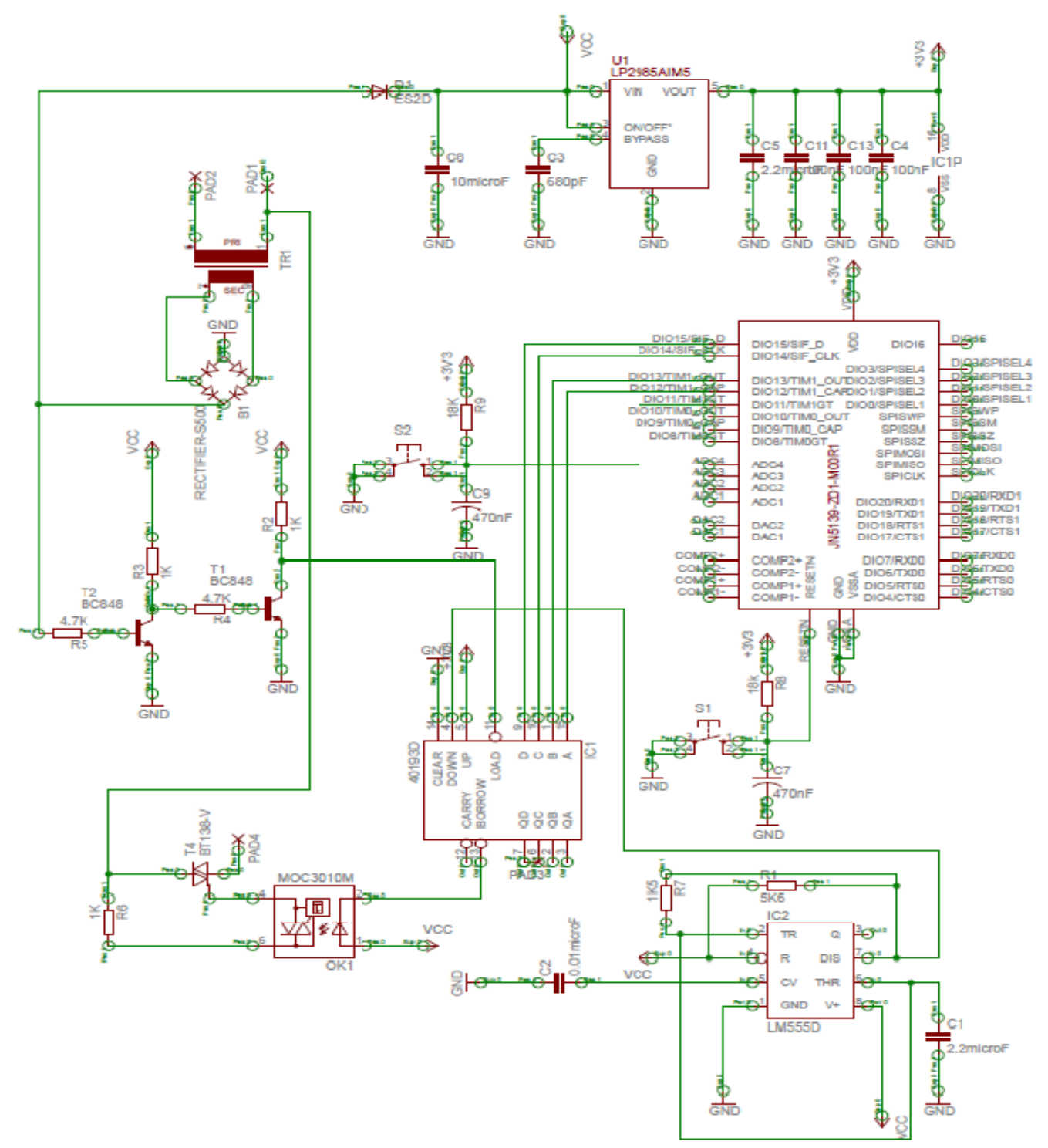

Figure 6. Dimmable light device schematic

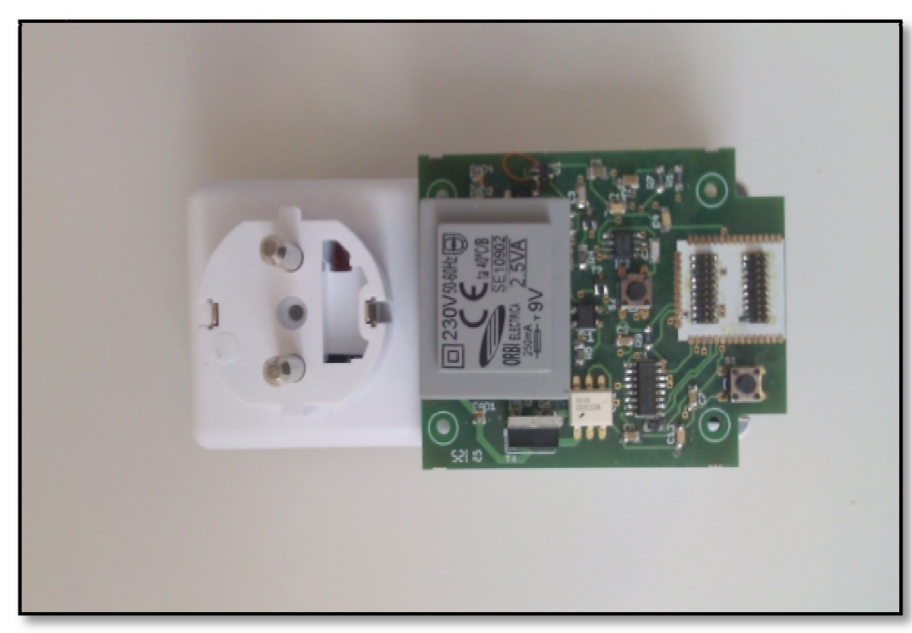

Figure 7. Dimmable light device PCB and case 
Apart from the schematic, the circuit has been routed into a PCB. The PCB can be shown in Figure 7. It is a PCB routed in two layers with most of its components in surface mount technology (SMD). The device is completed by encapsulating the PCB into a plug case. This case can be perfectly plugged into any home plug. The case is $15 \times 5 \times 3.5 \mathrm{~cm}$ (height, width, depth). It is not too high and future designs will be reduced to make it more comfortable.

Another device designed is a sensor that measures galvanic skin conductance (also called galvanic skin response - GSR). The GSR consists of applying a direct current constant voltage probe signal to the skin. In measuring skin conductance, one can distinguish two different principles: phasic and tonic. A tonic value stands for an activity that shows a certain degree of continuity over a time. The tonic component of skin conductance is the skin conductance level (SCL). SCL is obtained by measuring the total amount of skin conductance and is related to a person's overall arousal. In contrast to tonic values, a phasic value stands for the change in skin conductance within a short time period as a reaction towards a discrete stimulus. If a stimulus elicits an orienting response, the skin conductance rises for a certain time period and then returns to normal. This is called a skin conductance response $(\mathrm{SCR})$.

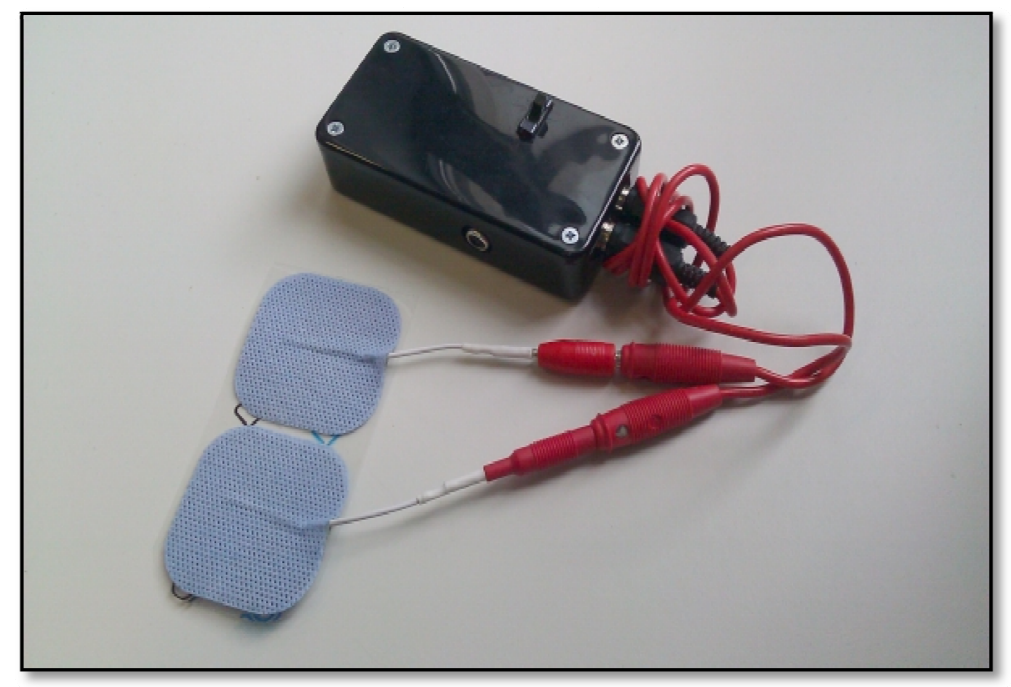

Figure 8. GSR prototype
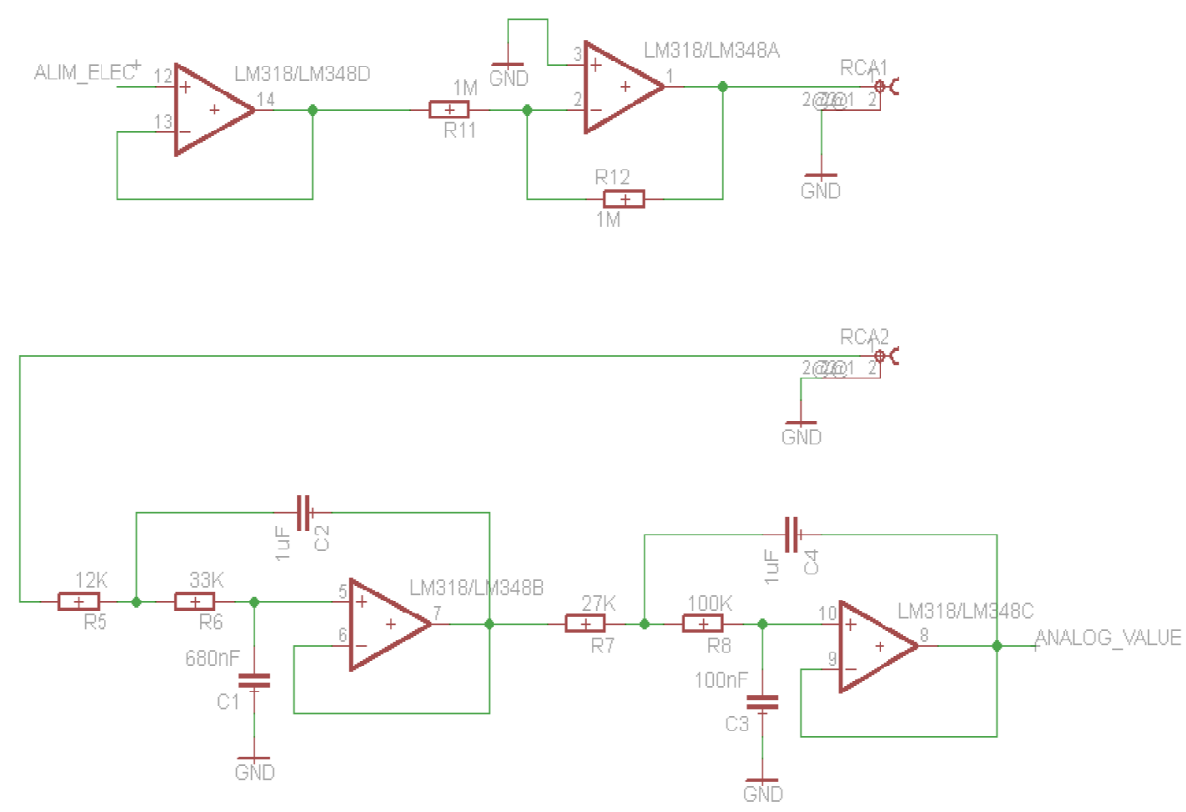

Figure 9. GSR device schematic 
The GSR prototype can be seen in Figure 8 and the device schematic is presented in Figure 9. It has two distinct parts: on one hand, the adaptation stage for the signal injected into the body. You cannot inject a lot of current in order to avoid electrocuting the patient. On the other hand, there is the filtering stage. The signal we need is within a small range of frequencies. More specifically, the signal is below $10 \mathrm{~Hz}$. Therefore, the second stage is the filter. This filter is of Butterworth type and fourth order. It has been designed with Sallen-Key topology.

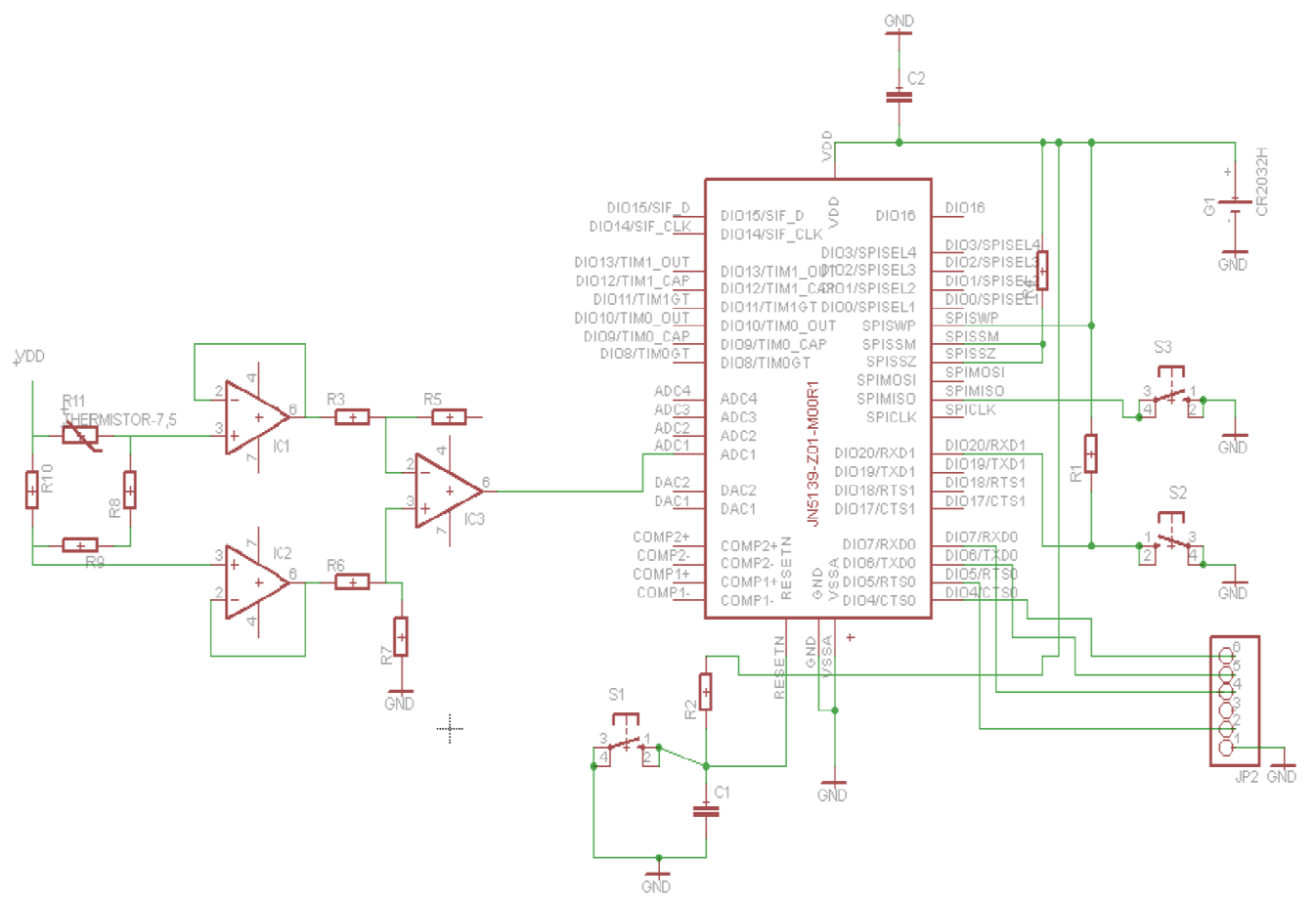

Figure 10. Thermometer schematic

And the last device is a thermometer, a general and massively used device to check body temperature. There are several types of body thermometers:

a) Mercury thermometer: it is a non-digital thermometer that uses the expansion of mercury contained in a glass tube. It is very cheap but the mercury is really harmful if the thermometer breaks.

b) Digital thermometer: it uses a thermistor to check the temperature. It is a variable resistance that changes with temperature. It is easy to read the temperature with an analog-to-digital converter and show it in a LCD panel.

c) Ear thermometer: it uses infrared technology, measuring the energy emitted by the tympanic membrane.

The type selected for the project is the digital one, using a thermistor to measure the temperature. It is easy to design and we can take advantage of the analog-to-digital converter included in the JN5148 microcontroller. The thermistor has been configured in a bridge as shown in the schematic of Figure 10. The thermometer has a button, attached to a DIO pin of the microcontroller. When it is pressed, the microcontroller wakes up and waits two minutes to stabilize the temperature. After those minutes, the converter samples the value and sends it through the network.

\section{Results}

\subsection{Autonomous Devices}

The following scenarios show the results concerning the autonomy of the devices and their application in a real scenario. Also presented are some captures from a sniffer which has been a great help during development.

- Scenario 1: Switch and light binding

In this first scenario, a simple switch and a simple light are presented. Both devices implement the On/Off cluster. The switch implements it as an output cluster and the light as an input cluster. In order to bind them, the switch and the light have to send a bind request to their parent (in this case, the coordinator) with the information on this cluster, and they receive a bind response from the coordinator. This request is sent by pushing one button on the 
device. When the binding has been carried out, if the same button is pressed on the switch device, the light device toggles one of its leds. In this case, it toggles because the switch is configured to send a toggle command. It can also be configured with two buttons, one to switch on and the other to switch off the light.

\begin{tabular}{|c|c|c|c|c|}
\hline Time & Source & Destination & Protocol & Info \\
\hline 29.797861 & $0 \times 189$ & $0 \times 0000$ & ZigBee ZDP & End Device Bind Request 5 rc: Jennic_00:00:0f:25:88, Target: 0xd189 \\
\hline 29.967818 & $0 \times 0000$ & $0 x d 189$ & zigBee ZDP & End Device Bind Response, status: success \\
\hline 31.537663 & $0 \times 8889$ & $0 \times 0000$ & ZigBee HA & Report Attributes, seq: 0 \\
\hline 32.409614 & $0 \times d 189$ & $0 \times 8889$ & ZigBee HA & command: $0 \times 02$, seq: 1 \\
\hline 32.425464 & $0 \times 8889$ & $0 \times 0000$ & ZigBee HA & Report Attributes, 5eq: 1 \\
\hline
\end{tabular}

Figure 11. Switch and Light binding capture

Figure 11 shows a capture from the Wireshark sniffer. Here can be seen the binding process between the devices with addresses 0xd189 and 0xe889 and the coordinator (0x0000). The toggle commands can be seen in the frames, checking they are ZigBee HA compliant.

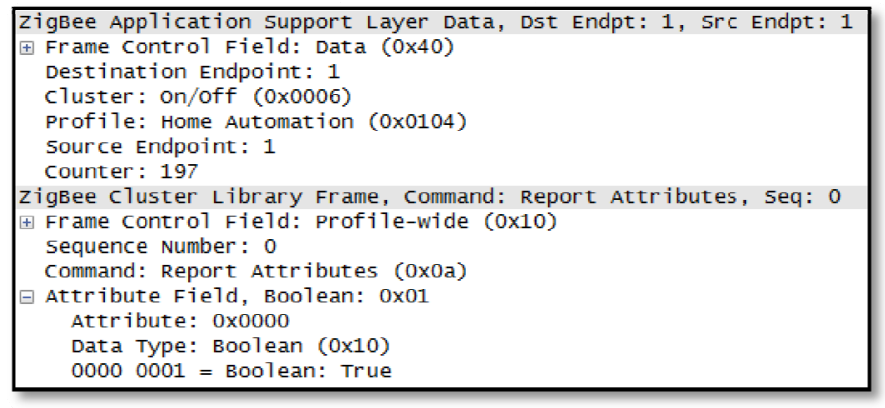

Figure 12. Report attribute frame

There are also some reports of attributes, because the light is configured to report changes in its attribute. This attribute is the value of the light, and in this case, as the switch device button has been pressed twice there are two reports: one to notify that the light has been switched on and the other notifying that it has been switched off. Figure 12 corresponds to the first report, when the light was switched on, so the report attribute (Boolean type) was set to true.

- Scenario 2: Dimmer and dimmable light binding

This second scenario is similar to previous one. The coordinator has to bind the dimmer switch with the dimmable light. The clusters implemented by them are the On/Off cluster and the Level Control cluster. As above, the switch has them as output clusters and the light as input clusters. They are both necessary: the on/off cluster provides the functionality to switch on or switch off the light. The level control one, instead, provides the functionality to change the light's level of brightness. It is necessary to do the binding process twice, one per cluster because the coordinator only makes one bind per cluster.

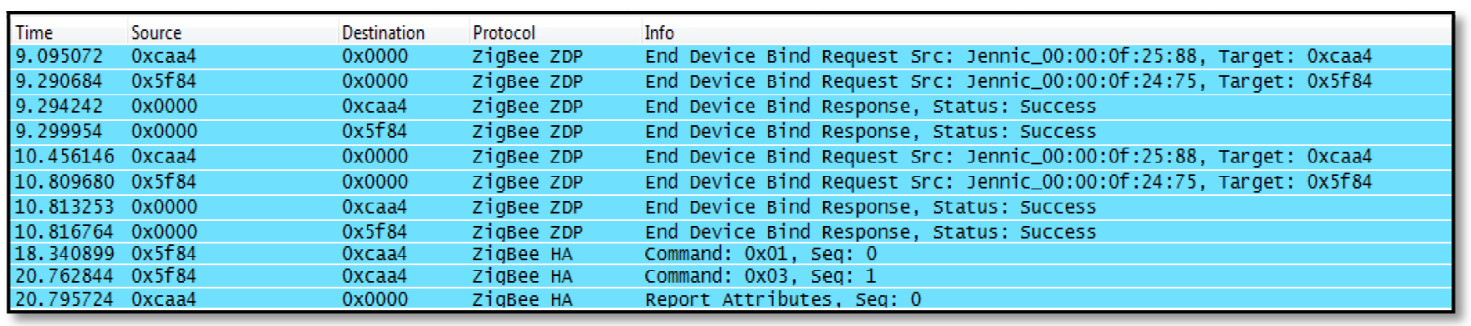

Figure 13. Dimmer and Dimmable Light binding capture

This binding process has been implemented by pressing the same button on the device. First, you press for a binding, and when it has been carried out, you press for the second one (see capture in Figure 13). 


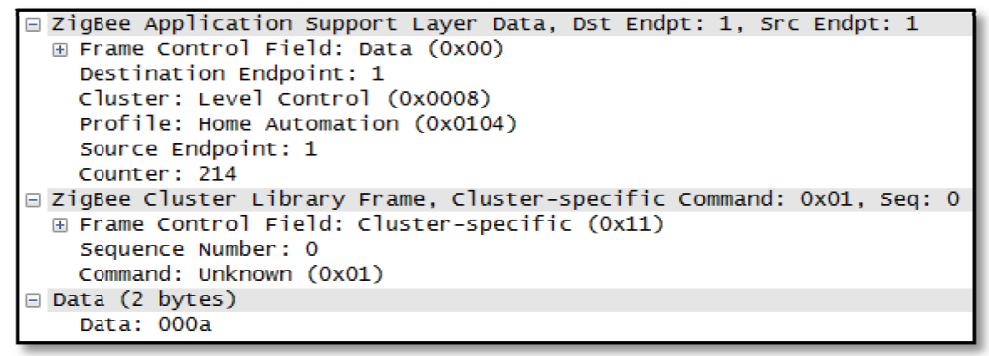

Figure 14. Level control move command

The following frames show the sending of move commands $(0 x 01)$ to increase the light brightness and the stop command (0x03), to halt the increases. There is only one report because it is configured to notify a change when it reaches a stable value; in this case, when the light receives the stop command. Figure 14 corresponds to the first command frame. It is a move command, so a mode (increase or decrease) and a step must be indicated (both bytes of data $0 \times 00$ and $0 \times 0 a$, respectively). This step is the increase or decrease per second -increase, in this case- in light brightness. It is stopped with the stop command $(0 \mathrm{x} 03)$.

- $\quad$ Scenario 3: Analog sensors

\begin{tabular}{|lllll|}
\hline Time & Source & Destination & Protocol & Info \\
17.259899 & $0 \times 3247$ & $0 \times 0000$ & ZigBee HC & Report Attributes, 5eq: 0 \\
22.267864 & $0 \times 3247$ & $0 \times 0000$ & ZigBee HC & Report Attributes, 5eq: 1 \\
27.277837 & $0 \times 3247$ & $0 \times 0000$ & ZigBee HC & Report Attributes, 5eq: 2 \\
\hline
\end{tabular}

Figure 15. Analog sensor capture

In this scenario there is no binding. The device has been developed to obtain samples of an analog sensor every five seconds. It samples and converts the value to digital and then sends it to the coordinator in a report frame, as is shown in Figure 15.

\subsection{Gateway: Controlling the Network from a PC}

The gateway scenarios show several functions that can be conducted from the serial port of a PC. The captures from the serial port are also presented.

- Scenario 1: Collecting information of devices

This scenario shows the initial need to develop user applications: to discover which devices are in the network and their information. In order to meet this need, there are several commands that can be sent to discover the endpoints, the clusters or the attributes of a device. The value of an attribute can be also requested from a device.

The notification of a new device reaches the serial port. The endpoints it has are asked for, and the response is one endpoint with $\mathrm{ID}=0 \mathrm{x} 01$. Then the simple descriptor of that endpoint is requested, which includes the profile associated to the endpoint, the device id and the input and output clusters. The response is that the endpoint $0 \mathrm{x} 01$ of the device with address 0xdc45 has associated the Home Automation Profile (0x0104). It is an On/Off Light (DeviceID $=0 \mathrm{x} 0100)$ and has two input clusters and two output clusters: the On/Off cluster (0x0006) and the Groups cluster (0x0004). After this, the device is asked for the attributes of the cluster 0x0006, and it has one attribute with ID=0x0000 and it is of Boolean type. Table 3 presents the commands exchanged through the serial port terminal while Figure 16 shows data sent by radio.

Table 3. Gateway commands in Scenario 1

\begin{tabular}{lll}
\hline DIR & PROTOCOL COMMAND & EXPLANATION \\
\hline IN & NDEV=DC45.00158D00000F2588.8E & New Device \\
OUT & GEPS $=$ dc45 & Get Endpoints of 0xdc45 \\
IN & SEPS $=$ DC45.01.01 & Get EPs Response \\
OUT & GSDS $=$ dc45.01 & Get Simple Descriptor \\
IN & SSDS $=$ DC45.01.0104.0100.00.02.0006.0004.02.0006.0004 & Get Simple Desc Response \\
OUT & ATDC $=$ dc45.01.01.0006.0000.02 & Attributes Discovery \\
IN & ATDR $=$ DC45.01.0006.01.0000.10 & Att Discovery Response \\
\hline
\end{tabular}




\begin{tabular}{|c|c|c|c|}
\hline $\begin{array}{l}\text { Source } \\
\text { 0xdc45 }\end{array}$ & $\begin{array}{l}\text { Destination } \\
\text { Broadcast }\end{array}$ & $\begin{array}{l}\text { Protocol } \\
\text { zigBee ZDP }\end{array}$ & $\begin{array}{l}\text { Info } \\
\text { Device Announcement, Device: Jennic_00:00:0f:25:88 }\end{array}$ \\
\hline $0 x d c 45$ & $0 \times 0000$ & ZigBee ZDP & Active Endpoint Response, Device: 0xdc45, status: Success \\
\hline $0 x d c 45$ & $0 \times 0000$ & ZigBee ZDP & Simple Descriptor Response, Device: 0xdc45, status: Success \\
\hline $0 \times 0000$ & $0 x d c 45$ & zigBee HA & Discover Attributes, Seq: 0 \\
\hline $0 \times d c 45$ & $0 \times 0000$ & zigBee HA & Discover Attributes Response, sea: 0 \\
\hline
\end{tabular}

Figure 16. Collecting information capture

\section{- Scenario 2: Unicast transmission}

The second scenario includes several commands that can be sent to each device as a unicast transmission. For example, we can switch on a light or increase the value of a dimmable light, or even ask for the present value of an analog sensor such as a galvanic skin response sensor. As in the previous scenario, and in Figure 17 shows the whole process from the serial port terminal and radio, respectively.

Table 4. Gateway commands in Scenario 2

\begin{tabular}{lll}
\hline DIR & PROTOCOL COMMAND & EXPLANATION \\
\hline IN & NDEV=1F7A.00158D00001108DF.8C & New device \\
IN & NDEV=FF12.00158D00000F2588.8E & New device \\
IN & NDEV=9F7F.00158D00000F2475.8E & New device \\
OUT & OOUC=ff12.01.01.02 & On Off Toggle command to 0xff12 \\
IN & ATRP=FF12.01.0006.0000.10.01 & Attribute Report \\
OUT & DMSU=9f7f.01.01.00.0a.0000 & Dimmer Move (UP) Step (0x0A) to 0x9f7f \\
IN & ATRP=9F7F.01.0008.0000.20.0A & Attribute Report \\
OUT & ATRD=1f7a.02.01.000c.0055 & Read Attribute 0x0055 of cluster 0x000c, of device \\
& & 0x1f7a \\
IN & ATRR=1F7A.01.000C.0055.39.000007C9 & Attribute Read Response \\
\hline
\end{tabular}

\begin{tabular}{|c|c|c|c|}
\hline Source & Destinution & Protocel & Info \\
\hline $0 x \perp f 7 a$ & Eroadcast & zigbee zOP & Device Announcement, Device: Jennic_00:00:11:08:df \\
\hline Oxff 12 & Broadcast & zigiete zOP & Device Announcement, Divice: Jennic_00:00:0f:25:88 \\
\hline Ox9f7f & Broadcast & zigeee zop & Device Announcement, Device: Jennic_00:00:0f :24:75 \\
\hline $0 \times 0000$ & $0 x f f 12$ & zigbee HA & Command: 0x02, Seq: 0 \\
\hline oxff 12 & $0 \times 0000$ & ziqueE HA & Report Attributes, seq: 0 \\
\hline $0 \times 0000$ & $0 \times 9 f 7 f$ & zigbee HA & Command: $0 \times 02$, seq: 1 \\
\hline oxgf7f & $0 \times 0000$ & Zigbee HA & Report Attributes, Seq: 0 \\
\hline $0 \times 0000$ & $0 \times 1 f 7 a$ & Zigbee HC & Read Ater fbutes, seq: 0 \\
\hline $0 \times 1 f 7 a$ & $0 \times 0000$ & ziqliee HC & Bead Attributes Response, sea: 0 \\
\hline
\end{tabular}

Figure 17. Unicast transmission capture

\section{- Scenario 3: Groups}

Another important feature is the possibility of adding devices to groups. It is possible to send a multicast frame so that all devices in that group will receive it. Beforehand, the devices should be added to a group. The protocol has some commands for adding, removing or discovering the groups of a device. This scenario combines the actions of the gateway and the action of the switch over a group bound to it.

As previously mentioned, the first step required is to add the devices to a group. In this case, there are two lights and a switch, and they all are added to the group ABCD. The lights include the groups in their groups tables and the switch in its binding table. The OOGR command sends a switch on command to the group. The lights added to that group change their light to on and then send a report with the Boolean attribute to true (0x01). The last two reports are caused by the switch. When the switch is pressed, it sends a toggle command to its bound devices. In this case, the bound device is a group, and both light devices switch off the light and send the consequent report. Table 5 and Figure 18, once again, present all the process. 
Table 5. Gateway commands in Scenario 3

\begin{tabular}{lll}
\hline DIR & PROTOCOL COMMAND & EXPLANATION \\
\hline IN & NDEV=A6C4.00158D00001108DF.8C & New device \\
IN & NDEV=A8FC.00158D00000F2475.8E & New device \\
IN & NDEV=8E10.00158D00000F2588.8E & New device \\
OUT & GRAD=a6c4.01.01.abcd & Add Group Request \\
IN & GRAR=A6C4.ABCD.00 & Add Group Response (Success=0x00) \\
OUT & GRAD=a8fc.01.01.abcd & Add Group Request \\
IN & GRAR=A8FC.ABCD.00 & Add Group Response (Success=0x00) \\
OUT & GRAD=8e10.01.01.abcd & Add Group Request \\
IN & GRAR=8E10.ABCD.00 & Add Group Response (Success=0x00) \\
OUT & OOGR=abcd.01.01 & Switch ON to group ABCD \\
IN & ATRP=D300.01.0006.0000.10.01 & Attribute Report \\
IN & ATRP=8E10.01.0006.0000.10.01 & Attribute Report \\
\hline
\end{tabular}

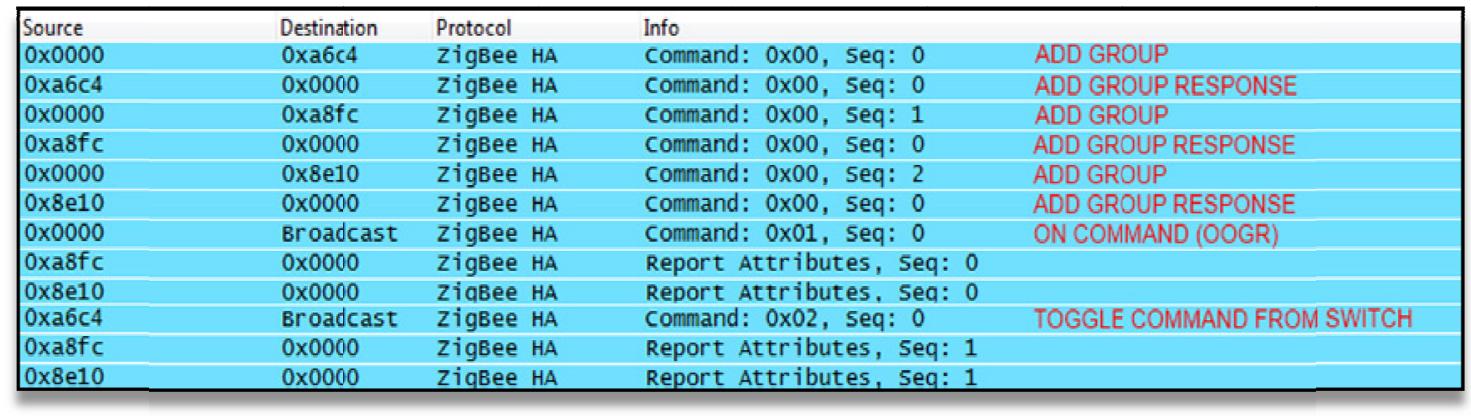

Figure 18. Groups capture

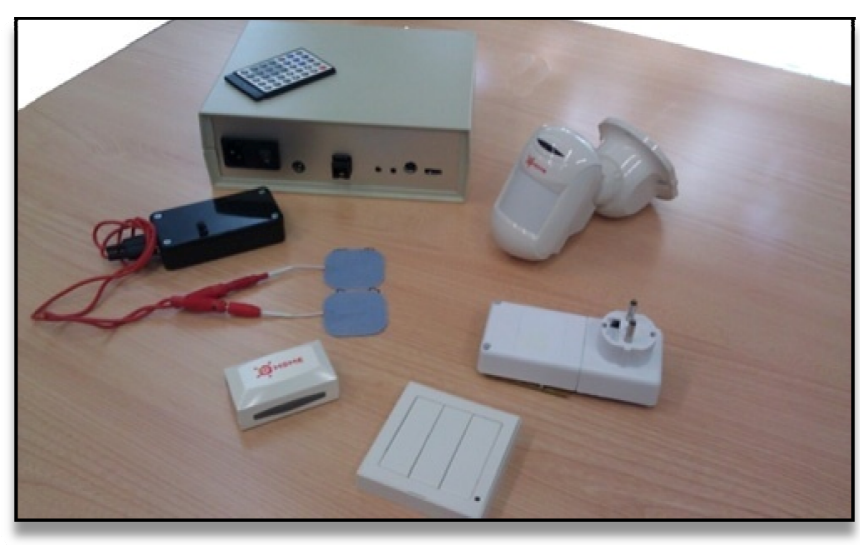

Figure 19. SetTop Box and some wireless devices

\subsection{System Integration}

All these devices have been integrated into the SetTop Box mentioned above. The complete system is shown in Figure 19, including the designed devices as well as other devices by different manufacturers. We have tested the end devices with our coordinator and others to verify that they are interoperable. In addition, network traffic captures have sniffed to verify that they meet the standard and to dismiss a lot of modules that have been acquired and are not ZCL compatible. Moreover, the SetTop Box includes an application software that enables the user to manage the wireless network while is watching TV. 


\subsection{Satisfaction Test}

We have also carried out several questionnaires with different people in terms of age and technological knowledge in order to analyze whether we are working in the right way. Although the questionnaire was simple, it was sufficient to test the system. The responses of the questionnaire had to be between the values of 1 and 5 (1 for "disagree", 5 for "strongly agree").

Among all the questions asked, the three most important are highlighted below. The users were asked if:

- The software application was easy to use.

- Creating the WSN was simple.

- The system is useful.

We interviewed some 25 people and the results are presented in Table 6 .

Table 6. Test results

\begin{tabular}{ll}
\hline Questions & Mean value \\
\hline Simplicity of User Application & 3.8 \\
Simplicity of creating the WSN & 3.6 \\
Utility and Usability & 4.35 \\
Complete System Mean value & $\mathbf{3 . 9 2}$ \\
\hline
\end{tabular}

We have come to the conclusion that people with higher technological knowledge are more critical than others with less expertise. Nevertheless, people believe that the project is extremely useful. According to the general mean value, the system is being developed in the right way but a great effort should be made to make it easier to use.

\section{Discussion}

As shown throughout the article, there is a growing need for health services. Life expectancy is increasing as well as the number of illnesses, which is why telehealth systems are highly demanded by users, professionals and governments. Our goal as developers is to design systems that help people without involving major changes in their lifestyle or without a long training being needed to use it. To avoid these two aspects, adaptive systems are needed. This means having systems that are self-configuring in order to require as little user intervention as possible, thus enhancing its ease of use, one of the goals of this project.

Apart from this feature, another important goal was to develop a system based on a standard, thereby promoting interoperability between devices by different manufacturers. Firstly, a preliminary state-of-the-art analysis of wireless technologies was carried out until ZigBee was selected. ZigBee is a technology that is taking off slowly, step by step. Its entry into the market is hard because it is open standard. There are other protocols that are owned by manufacturers of consumer electronics causing their prompt take-off in the market. Even so, ZigBee has the skills and features necessary for a wide deployment of sensors in a network regardless of the application type (home automation, security, healthcare, safety...).

As presented in the results section, specific and general application features have been developed. These features are: binding, in order to decentralize network management and allow independent operation of devices, and the ability to create groups of devices to manage them as a group rather than individually.

Despite enabling independent operation, the system can be controlled by a user application. The idea is to have an intuitive and easy-to-use application that manages the network. For this reason, a gateway has been also developed allowing information exchange between the network coordinator and any embedded device. A user interface can be designed to run on any platform or operative system and be able to connect with the network through a serial communication port. This provides many possibilities as the user application can use different technologies. In this case, the embedded device could act as a bridge between the sensor network based on ZigBee and the other networks. The gateway developed is oriented to the described scenarios but can easily be expanded or customized to fit in any design.

There are similar types of systems on the market like the one presented in this article. One example is the AlertMe system, which is a complete product for home automation and control. It has sensors that detect if a window or a door has been opened, devices to turn on electric appliances, motion sensors, and so on. It is a scalable system throughout its range of products, but it offers no interoperability with other devices. It is a closed 
product, based on the first ZigBee release. So if you need a particular device and it is not developed by the manufacturer, you cannot integrate a device from another manufacturer in the AlertMe system. Another company with similar ZigBee compliant products is Simplehomenet, a company experienced in different technologies such as X10, Insteon or ZigBee. It markets gateways of these technologies, but they have some products that still have not been adapted to ZigBee. However, products that are already adapted meet the latest ZigBee release.

There are not a great number of products on the market. There are no complete or customized systems that can interoperate with other devices, or systems that anyone can install at home regardless of their technological knowledge. New technologies are increasingly appearing, not only more, but better ones. This prevents the development of systems with previous technologies and they become unfinished and unsupported systems. We suggest that more technologies are not needed and we have to exploit existing ones. In addition, this fast leaping from one technology to another does not provide sufficient time for companies to recover their investment, causing a lack of interoperable systems that requires more effort. And after all, this is what is needed to create value-added services.

The expansion of profiles used in the devices is proposed as a future line of work. Other future work could be the design of new devices oriented to healthcare, or the adaptation of designed ones to the ZigBee Pro standard. And finally, as regards software development, we propose the design of an intuitive and easy-to-use user application. The application could be adaptable, depending on the collective to which it is oriented.

\section{Conclusions}

As a final reflection, below is a review of the aims proposed at the beginning of this article that have been fulfilled.

As regards the implementation of ZigBee in application devices, several scenarios have been presented as a result of the developments carried out. These scenarios present, in a clear and simple way, everything developed in the devices to attain their use in real environments. These developments are the base or the mainstay for future development of specific applications. It is the beginning of a long road towards a complete system that provides value-added services based on a unique technology.

Likewise, the development of a gateway making it possible to connect the system to different systems has been successfully implemented. The gateway can be extended depending on the level of specificity needed, thus enabling easier use in more specific future applications.

The scenarios presented above are based on some tests performed with several devices of both home automation and health profiles. Since it is a work in progress, we have started studying ZigBee standard by developing general devices (such as switches and dimmers). It is a simple but effective way of studying the standard in order to establish knowledge.

Health sensors and devices are being designed in a parallel way to be integrated in the system as soon as possible. So, the work presented is the basis of the complete system and the progress of a long future work including integration of new health devices as well as an user application to control the whole network.

The main aim is to produce a complete system for future deployment in patients' homes.

\section{References}

Akematsu, Y., \& Tsuji, M. (2009). Economic effect of eHealth: Focusing on the reduction of days spent for treatment. 11th International Conference on e-Health Networking, Applications and Services. pp. 14-20.

Cabral, J. J., \& Kim, Y. (1996). Multimedia systems for telemedicine and their communications requirements. IEEE Communications Magazine, 34, pp. 20-27. http://dx.doi.org/10.1109/35.526884

Chadwick, P. (2007). Regulations and Standards for Wireless applications in eHealth. 29th Annual International Conference of the IEEE Engineering in Medicine and Biology Society. pp. 6170-6173.

Chien, C., Xu, Z., \& Molloy, S. (2010). Topics in integrated circuits for communications. IEEE Communications Magazine , 48, 74. http://dx.doi.org/10.1109/MCOM.2010.5439079

Chuang-Chien Chiu, T.-W. S.-C.-C.-Y. (2006). A Wearable e-Health System with Multi-functional Physiological Measurement. World Congress on Medical Physics and Biomedical Engineering, 14, 429-432.

De, M. G. (2005). Trends in Health Telematics: Electronic Health Records in an Intelligent and Communicating Environment. Universal Access in Health Telematics. Lecture Notes in Computer Science, 3041, pp. 1393-1397.

Detmer, D., Bloomrosen, M., Raymond, B., \& Tang, P. (2008). Integrated Personal Health Records: 
Transformative Tools for Consumer-Centric Care. BMC Medical Informatics and Decision Making, 8, 1-14. http://dx.doi.org/10.1186/1472-6947-8-45

Farshchi, S., Pesterev, A., Nuyujukian, P., Mody, I., Judy, J., \& Bi, F. (2007). An Embedded Sensor/System Architecture for Remote Biological Monitoring. IEEE Transactions on Information Technology in Biomedicine , 11, 611-618. http://dx.doi.org/10.1109/TITB.2007.897600

Gibbons, M. (2008). Disparities and eHealth: Achieving the promise and the potential. In Gibbons, \& M. Christopher, eHealth Solutions for Healthcare Disparities. New York: Springer. pp. 154-164.

Gomez, C., \& Paradells, J. (2010). Wireless home automation networks: A survey of architectures and technologies. IEEE Communications http://dx.doi.org/10.1109/MCOM.2010.5473869

Hine, N., Petersen, F., Pluke, M., \& Sund, T. (2008). Standardization work on personalized eHealth systems. 30th Annual International Conference of the IEEE Engineering in Medicine and Biology Society. pp. 1518-1520.

Hui, Z., Yanting, G., \& Xue, W. (2010). Theoretical and Empirical Study on the Relationship between Household Health Investment and Government's Public Health Investment. International Conference on Management and Service Science. pp. 1-4.

Hwang, K., In, J., Park, N., \& Eom, D. (2003). A design and implementation of wireless sensor gateway for efficient querying and managing through World Wide Web. IEEE Transactions on Consumer Electronics, 49, 1090-1097. http://dx.doi.org/10.1109/TCE.2003.1261201

Iakovidis, I., Le, D. O., \& Karp, P. (2007). Biomedical Engineering and eHealth min Europe - Outcomes and Challenges of Past and Current EU Research Programs. IEEE Engineering in Medicine and Biology Magazine , 26, 26-28. http://dx.doi.org/10.1109/MEMB.2007.364925

Iluyemi, A., \& Briggs, J. (2009). eHealth and Global Health: Investments Opportunities and Challenges for Industry in Developing Countries. Electronic Healthcare. Lecture Notes of the Institute for Computer Sciences, Social Informatics and Telecommunications Engineering , 1, 182-185.

Krol, M. (1997). Telemedicine. IEEE Potentials, 16, 29-31. http://dx.doi.org/10.1109/45.624339

Bandyopadhyay, L. K., S. C. (2010). ZigBee Technology: A Unique Wireless Sensor Networking Solution. Wireless Communication in Underground mines, 153-174.

Le, M. B., \& Ray, P. (2009). Issues en E-Health Cost Impact Assessment. World Congress on Medical Physics and Biomedical Engineering, 25/12, 223-226. IFMBE Proceedings.

Lymberis, A., \& Dittmar, A. (2007). Advanced Wearable Health Systems and Applications - Research and Development Efforts in the European Union. IEEE Engineering in Medicine and Biology Magazine , 26, 29-33. http://dx.doi.org/10.1109/MEMB.2007.364926

Mauksch, M. W. (1987.). The Aging Population: The New Growth Market. Ageing International, 14(2), 23-24. http://dx.doi.org/10.1007/BF02999842

McAdams, E., Krupaviciute, A., Gehin, C., Dittmar, A., Delhomme, G., Rubel, P., ... McLaughlin, J. (2011). Applications to Medical Diagnostics/Monitoring. In Bonfiglio, A., \& Rossi, D. d. Wearable Monitoring Systems. Springer.

Nitzkin, J. (1996). Technology and health care-driving costs up, not down. IEEE Technology and Society Magazine, 15, 40-45. http://dx.doi.org/10.1109/44.536300

Paul, D., Pearlson, K., \& McDaniel, R. J. (2009). Assessing technological barriers to telemedicine: technology-management implications. IEEE Transactions on Engineering Management , 46, 279-288. http://dx.doi.org/10.1109/17.775280

Piniewski, B., Muskens, J., Estevez, L., Carroll, R., \& Cnossen, R. (2010). Empowering Healthcare Patients with Smart Technology. IEEE Computer Magazine, 27-34.

Poon, C., \& Zhang, Y. T. (2008). Perspectives on High Technologies for Low-Cost Healthcare. IEEE Engineering in Medicine and Biology Magazine, 27, 42-47. http://dx.doi.org/10.1109/MEMB.2008.923955

Sheng, O., Hu, P. H., Wei, C. P., \& Ma, P. C. (1999). Organizational management of telemedicine technology: conquering time and space boundaries in health care services. IEEE Transactions on Engineering Management , 46, 265-278. http://dx.doi.org/10.1109/17.775279 
Song, G., Zhou, Y., Zhang, W., \& Song, A. (2008). A multi-interface gateway architecture for home automation networks. IEEE Transactions on Consumer Electronics, 54, 1110-1113. http://dx.doi.org/10.1109/TCE.2008.4637595

Sudhahar, S., Vatsalan, D., Wijethilake, D., Wickramasinghe, Y., Arunathilake, S., Chapman, K., et al. (2010). Enhancing Rural Healthcare in Emerging Countries through an eHealth Solution. ETELEMED '10. Second International Conference on eHealth, Telemedicine, and Social Medicine. pp. 23-29.

Vasilyeva, E., Pechenizkiy, M., \& Puuronen, S. (2005). Towards the framework of adaptive user interfaces for eHealth. 18th IEEE Symposium on Computer-Based Medical Systems. pp. 139-144. http://dx.doi.org/10.1109/CBMS.2005.101

Wells, P. (2002). Can technology reduce health care costs? Engineering Management Journal, 12, $194-200$. http://dx.doi.org/10.1049/em:20020410

Yan, H., Huo, H., Xu, Y., \& Gidlund, M. (2010). Wireless sensor network based E-health system: implementation and experimental results. IEEE Transactions on Consumer Electronics, 56, 2288-2295. http://dx.doi.org/10.1109/TCE.2010.5681102 